\title{
Production of pre-sexed racing camel offspring using embryo biopsy and detection of camelid sex specific Polynucleic acid sequences
}

\author{
Tinson $^{1}$ A.H., Harrison ${ }^{2}$ B., King ${ }^{2}$ B., Singh ${ }^{1}$ K., $\operatorname{Wade}^{1}$ N. and Reed K ${ }^{3}$ \\ ${ }^{1}$ Embryo Transfer Research Centre Racing Camels, H.H. The Crown Prince Sheikh \\ Khalifa bin Zayed Al Nahyan P.O. Box 17292 Al-Ain United Arab Emirates \\ ${ }^{2}$ Camelot BioScience Qld. Australia, \\ ${ }^{3}$ Queensland Agriculture and Biotechnology Center, Australia
}

\begin{abstract}
Breeding Racing Camels is a slow process given their long gestation period, poor yearly calving rate and the long racing life enjoyed by the champion female camels. Embryo transfer in the dromedary camel has sped up the process of quality genetic replacement of retired race camels allowing up to 12 calves to be carried by surrogates as offspring from one set of genetic parents. The successful pre-sexing of multiple embryos prior to their transfer offers further flexibility to a camel embryo transfer programme. The technique involves micromanipulation and biopsy of the embryo followed by PCR (polymerase chain reaction) for the detection of the sex specific sequences. With pregnancy rates similar to that of non-biopsied embryos the technique allows for the more efficient use of surrogate mothers, specific selection for the more valuable female offspring and a commercial opportunity to produce guaranteed sex offspring. The technology also has an important potential application in the production of superior camel milking herds as well as protection of endangered camel populations where increased female numbers are crucial for success. A diagnostic "sex test" on an embryo is also the start of what might one day be a series of genetic tests on camel embryos. We routinely test the camels blood prior to racing but soon we might have routine genetic tests prior to breeding that could relate to speed, milk production or even disease resistance.
\end{abstract}

Key words: polynucleotide, pre-sexed, biopsy, embryo and racing camel.

\section{Introduction}

Many sectors of the various camelid industries prefer a prepondereance of animals of one sex. This maybe for reasons of reproductive potential, heritability of particular traits, tractability, performance, stature and physique and appearance. In the case of the dromedary camel the lucrative racing industry of the Middle-East has a preference for females due to their speed, prize potential and racing longevity. In the case of milking camels such as the black Saudi Arabian dromedary there is obviously a preference for female camels. In India where draft animals are often prized the male is more sought after offspring. The ability to determine the sex of a foetus is advantageous since it allows optimal management and evaluation of pregnancies. In the case of camels the ultrasonic determination of a foetuses sex to date has been difficult to achieve. In the case of assisted reproductive technologies such as embyro transfer determining the sex of an embryo is even more advantageous since it allows the sex of potential progeny to be predetermined. In sheep and cattle if the technique is combined with artificial twinning by means of embryo bisection (Herr, 1991) it further allows enhanced propagation of the desired sex without reduction in the total number of potential progeny. With top female racing camels fetching prices in excess of \$1 million US the applications of these techniques in racing camels make obvious economic sense. The primary sex of camelid species, as in the overwhelming majority of mammalian species, is determined by the presence or absence of the entire $Y$ chromosome or a functional portion thereof (Ford et al. 1959; Jacobs and Strong, 1959). The essential portion is a gene known as SRY that is responsible for initiating testis differentiation (Sinclair et al. 1990. 


\section{A. H. Tinson et al}

Koopman et al. 1991). Secretions of the resultant testis have a dominant effect on the development of secondary sex characters (Jost, 1973). The sex or presumptive sex of an individual camel can thus be determined by analysis for DNA sequences that are associated uniquely with the camel Y chromosome.

\section{Materials and Methods}

In March 1998, nine female retired racing camels were selected to be donors for the embryo collection along with thirtyfive recipient camels. All camels were between 6 and 12 yrs old and had previously had calves. The donors and recipients were prepared as previously described (McKinnon et al. 1994). Tinson 1998) and 7 of the 9 donors responded with good superovulatory responses and were subsequently flushed seven days after they were mated naturally to ex-racing male camels. Four of the donors flushed, produced 23 embryos and the other three failed to give anything. The 23 embryos consisted of 17 hatched blastocysts and 6 expanded blastocysts. The recovered embryos were washed and held in Vigro Holding solution (AB Tech USA) in preparation for biopsy.

A Transferman Micromanipulation System (Eppendorf, Germany) attached to an Leica DMIRB/E Inverted Microscope (Leica Germany) was used to perform the biopsies. The microscope is fitted with LMS (light modulation system) based on the Hoffman modulation contrast principle to allow a more three dimensional view of the subject. Embryos were placed individually in small circular petri dishes (Falcon No351006, 50x9mm, USA) in a drop of Vigro Splitting Plus solution (ABTech USA) under oil to allow the manipulations to take place. The 23 embryos were divided into 2 groups, 17 had a small biopsy of an estimated 5-20 cells from the trophoblastic outerlayer of the embryos and the remaining 4 embryos were initially split into demi-embryos (in the hope of producing twins) and also biopsied at the same time. The biopsies were performed by attaching an $\mathrm{AB}$ Tech Twinning blade (ABTech USA) to the Eppendorf Transferman robotic arm with the blades fine movement controlled by the joy-stick control. The biopsies were recovered from the splitting solution using the Drummond pipette (Drummond Scientific PA, USA) with 2 ul of Vigro retrival solution by expelling half the volume of the Vigro retrieval media over the biopsy and biopsied embryo, then drawing the biopsy into the Drummond pipette. The mixture of retrieval solution and splitting solution containing the biopsy was then transferred to a 20ul Corbett research thermocycling capillary containing $2 \mathrm{ul}$ of lysis buffer. The biopsied embryos and demi-embryos were then recovered from the splitting drop transferred to labeled 4-well IVF plates (Falcon No3654 US) containing Vigro Holding solution in preparation for the later transfers to surrogates. The biopsy was incubated at $55^{\circ} \mathrm{C}$ for 10 minutes, $98{ }^{\circ} \mathrm{C}$ for 8 minutes and then place on ice. Then $16 \mathrm{ul}$ of a duplex PCR master mix including male specific primers based on the patented camelid sex male specific polynucleotide sequence (Patent Application NoGCC/P/99/125) and camelid specific autosomal control primers was then added to the lysed camel embryos biopsies. The capillaries were then place into a Corbett Research FTS-4000 Capillary Thermocycler and subjected to a thermocycling profile of $95^{\circ} \mathrm{C}$ for 20 minutes, then 8 cycles of $94{ }^{\circ} \mathrm{C}$ for 15 seconds and $68{ }^{\circ} \mathrm{C}$ for $30 \mathrm{sec}$ then $72{ }^{\circ} \mathrm{C}$ for 30 seconds followed by a further 47 cycles of $94{ }^{\circ} \mathrm{C}$ for 15 seconds and $72{ }^{\circ} \mathrm{C}$ for 35 seconds finally followed by a single cycle of $72{ }^{\circ} \mathrm{C}$ for 2 minutes then the samples were removed and cooled by ice. The 
complete contents of the capillary were then loaded onto a $2 \%$ agarose gel in $1 \mathrm{xTAE}$ buffer and electrophoresed for 30 minutes at 130 volts, the ethidium bromide stained gel was then visualized under UV radiation. No bands are observed in the absence of camelid DNA, whereas a single 230 base pair band is observed in the presence of male and female DNA (internal autosomal control) and if the male DNA is present there is another 470 base pair band present. Once the results of the sexing were determined the sexed embryos were transferred to the synchronized surrogates to establish pregnancy (McKinnon et al 1994, Tinson 1998).

The sequences were determined from cloned inserts of recombinant plasmid that were derived from male specific products of RAPD PCR on pools of male or female camelid DNA. Sex specificity was confirmed by Southern Blot Hybridization analysis and PCR on a panel of individual male and female camelid DNA, using probes and primers based on the CAMY.11 sequence (G.C.C.Patent Applic. NoGCC/P/99/125). More recently in 1999 significant improvement on this protocol was developed with the adaption of the assay to a real time PCR format using a Corbett Research Rotor Gene Real-Time PCR instrument (Corbett Research,Australia) with the following modification to the protocol. A thin walled $0.2 \mathrm{ml}$ PCR tube replaced the PCR capillary and the design of the design of the male specific (based on the male specific sequence) and autosomal primers and probes to enable real time detection of the amplified product. The Corbett Machine is an advanced centrifugal, real time DNA amplification system. It features 4 separate light sources with 6 separate detection filters. The system can detect up to 4 separate probes at once and no software algorithm is required to analyse the data. The rotary nature of the machine allows for very short hold times as temperature equilibrium is minimal and works in a range of 25-99 degrees centigrade.

The Rotogene software integrates the PCR machine and a Hewett Packard computer to give a read out of the detection of the $\mathrm{Y}$ specific sequences as well as the presence of autosomal DNA. This system was trialed in April 1999 on the end of a routine embryo collection. In January 2000, 3 embryos, 3 expanded blastocysts from a 7-day flush were biopsied using the technique described above. The biopsies were analyzed using the Corbett Rotogene PCR and the embryos subsequently transferred.

\section{Results}

Biopsies were performed and analyzed and both groups of biopsied embryos were transferred individually to the respective surrogates. Of the 23 embryos biopsied remarkably 16 were detected as female and 7 only were male. The 19 "biopsy only" embryos resulted in the establishment of 9 pregnancies, which continued to term with the world's first pre-sexed embryo transfer calf born on 3/4/99 as predicted a female with a gestation of 360 days. The 4 "splitbiopsied embryos were transferred as 8 demi-embryos but only resulted in the establishment of a single pregnancy and thus no twins were created. The last 10 presexed births occurred on the 24/4/99 with the longest gestation being 381 days. Out of the live births of 10 offspring all the predicted sexes were correct except one where a predicted female birth resulted in a male birth which was due to a handling mistake.

In January 2000 the sexing results with the Rotogene resulted in 2 of the embryos diagnosed as male and one female. The transfer of the sexed embryos resulted in 2 from 3 pregnancies $(66 \%)$ with both calves 


\section{A. H. Tinson et al}

born 390 days later with normal deliveries and correct sex predictions. All births were within the normal gestation range for dromedary camels (Tibary and Anouassi 1997, Manefield and Tinson 1996).

\section{Discussion}

The sexing of the pregnant foetus in the camel at around 60 days as is done in the horse (McKinnon 1997) has proved difficult in camels. Also once pregnancy is established then one has no further influence on the outcome of sex, whereas with pre-sexing of the embryos one can elect to transfer or not, depending on the result of birth that is required. The ability to use polynucleotide sequences for sexing provides the potential advantages and disadvantages are listed in Table 1.

The term "polymerase chain reaction" or "PCR" refers to the procedure where minute amounts of a specific piece of nucleic acid (DNA or RNA) is amplified as described in (Mullis et al. 1986). Sequence information from the ends of regions of interest need to be available such that oligonucleotide primers can be designed so that the primers will be identical in sequence or similar in sequence to opposite strands of the template to be amplified. The designed sex specific sequence hybridizes specifically to the camel Y-chromosome. Hybridization specifically to the $Y$ chromosome refers to the polynucleotide hybridizing to a single or repeated sequence which is present exclusively on the camel Y chromosome or in a substantially greater copy number than is present elsewhere in the camel genome. This work was in many ways a direct follow on from earlier sexing work done in ruminants by Reed and coworkers in the 1980's (Reed et al., 1986).

Table 1. Advantages and Disadvantages of Sexing Camel Embryos Prior to Transfer

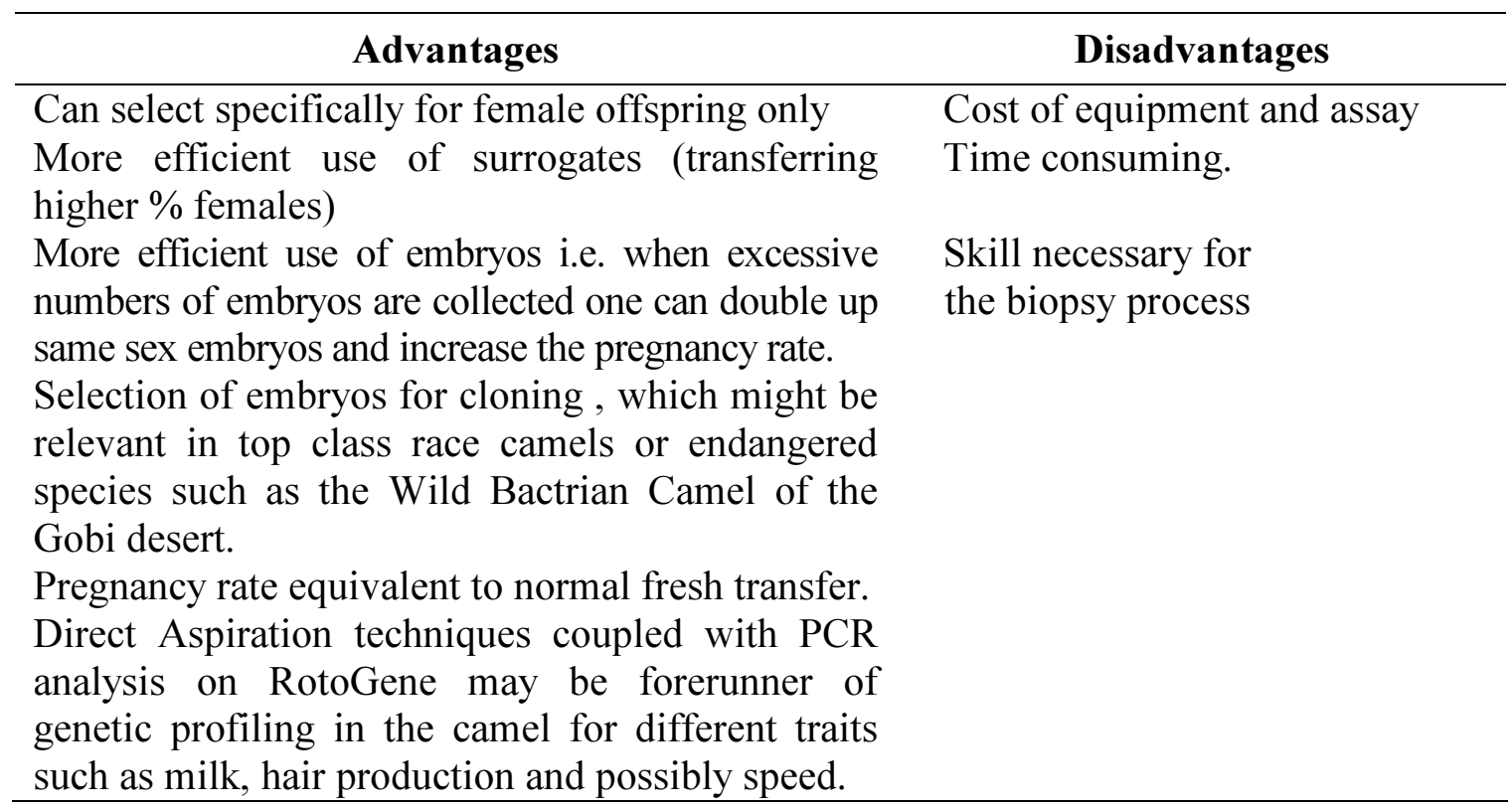

In a diagnostic assay for genetic sex, no detectable product of PCR amplification from male-specific primers may result not from a female sample but from PCR failure or loss of sample. For this reason a duplex PCR assay was developed in which a 200 base pair fragment of an autosomal repeated sequence was simplified simultaneously with the Y-specific target. The Rotogene is able to simultaneously detect both reactions to confirm a reliable test has been carried out. The Y-chromosomal specific element 
CY.AM11 has been shown to be present in all camelid species tested which included Dromedary and Bactrian Camels, llamas and alpacas (Harrison, 2001). The less domesticated guanaco is a part of the Llama genus and is expected to contain the same sequence. Wild vicunas have to date been unable to have been sampled.

The camel embryo at the hatched and expanded blastocyst stage appears to be robust when it comes to micromanipulation. The pregnancy rate of 9/19 for the biopsy only embryos compares well with other reported pregnancy rates in camels (McKinnon et al 1994, Tibary and Anouassi 1997, Tinson 1998). Previously the authors had experimented with biopsy of 9-day megablasts and managed to attain over $70 \%$ pregnancy rate in sectioned transferred embryos. It would seem the camel embryo is much more comfortable with micromanipulation than freezing given previous freezing results of $20 \%$ and less (Manefield and Tinson 1996). The problem with the twinning appeared to be the positioning of the embryo to allow correct bisection of the inner cell mass when using hatched blastocysts. Electrostatic attraction of embryos to the floor of the dish doesn't allow the accurate positioning needed to perform the procedure successfully. Recent development of a new micropipette has allowed better results with twinning (Tinson et al. 2001). The development of the analysis of the assay with the Rotogene now allows for lower flush to transfer times at the time of sexing. It is expected that with the new program the ramping time should come down to under 1 hour with larger biopsies being positive within 27 cycles (Harrison, 2001).

The error in one of the calves was probably not a bad thing in the course of the development of the technology in that it led to evaluation of the sampling and handling process post experiment. Subsequent work by Harrison (2001) has led to the development of a Direct Aspiration Controls System, which with a special glass needle and collection tube will eliminate handling errors, and the potential of post PCR product contamination when dealing with single cell biopsies. It will be possible to take these small biopsies with the system using unprecedented fine control and streamlining of testing procedures. Double manipulations such as biopsy and twinning will also become a better prospect under these conditions.

\section{Conclusions}

The determination of the sex of the camel embryo is a viable procedure that gives results comparable to fresh embryo transfer without manipulation and offers the flexibility to use the embryos and surrogates more efficiently when doing embryo transfer in camels. This is particularly evident when selecting sex for breeding racing or milking camels. The techniques have also led to a better understanding of how to approach twinning in camels as well as more accurate genetic testing principles in general by the evolution of the sampling process currently underway. The "sex test" looks set to be just the first in a series of tests that will be able to apply to the camel embryo. Just as a blood test is routine, a DNA screening much like is already happening at human IVF clinics could be developed for camels. A kit similar to that available for cattle cold be envisaged in the future and more efficient testing of samples would also lead to a decrease in the cost of the work and make it applicable to a wider range of animals. The conservation projects currently going on with the Wild Endangered Bactrian Camel in the Gobi desert will also have the potential to benefit from the sexing of its embryos so that the female groups can be increased in number at a more rapid rate when applying assisted reproductive techniques. 


\section{References}

Ford, C.E., K. W. Jones, P. E. Polani, de Almeida, J.C. and Briggs J.H., 1959. A sex chromosome anomaly in a case of gonadal dygenesis (Turners syndrome). Lancet I:711-713

Herr C., N. Holt, U. Pietrzak, Old, K. and Reed, K., 1990. Increased number of pregnancies per collected embryo by bisection of blastocyst stage ovine embryos. Theriogenology 33:244.

Herr, C. M. and K. C. Reed, 1991. Micromanipulation of bovine embryos for sex determination. Theriogenology $35: 45-54$.

Harrison B. 2001. The y-chromosomal specific element CY.AM 11 present in all camelid species. Personal communication.

Jacobs, P. A. and J. A. Strong, 1959. A case of human intersexuality having a possible XXY sex determining mechanism. Nature 183:302-303.

Jost, A., B. Vigier, J. Prepin and J. P. Perchellet, 1973. Studies on sex differentiation in mammals. Rec.Prog. Horm. Res. 29:1-41.

Koopman P., J. Gubbay, N. Vivian, P. Goodfellow and R. Lovell-Badge, 1991 Male development of chromosomally female mice transgenic for Sry. Nature 351:117-121.

Manefield G. W. and A. H. Tinson, 1996. "Camels- A Compendium", Vade Mecum SeriesC No 22 Post Grad. Foundation Sydney University.1996 372 pages.

McKinnon A. O., A. H. Tinson and G. Nation. Embryo Transfer in Dromedary Camels. Theriogenology 41:145-150, 1994.
Mullis, K., F. Faloona, S. Scharf, R. Saiki, G. T. Horn and H. Erlich, 1986. Specific enzymatic amplification of DNA in vitro: the polymerase chain reaction. Cold Spr. Harb. Symp. Quant. Biol. 51:263-273.

Reed K.C., M. E. Mathews and Jones, M.A.S., 1986. Sex Determination in ruminants using Y-chromosome specific polynucleotides. PCT AU87/00254, WO88/01300.

Sinclair A. H., P. Berta, M. S. Palmer, J. R. Hawkins, B. L. Griffiths, M. J. Smith, J. W. Foster, A. M. Frischauf, R. Lovell-Badge and P. N. Goodfellow, 1990. A gene from the human sexdetermining region encoding a protein with homology to a conserved DNAbinding motif. Nature 346:240-244.

Tiersch T. R., J. M. Mitchell and S. S. Wachtel, 1991. Studies on the phylogenetic conservation of SRY gene. Hum. Genet. 87: 571-573.

Tibary and Anouassi 1997 Theriogenology in Camelids. Abu Dhabi Publishing pp405-406.

Tinson A. H., K. Singh and K. S. Kuhad. Large Scale Management of Camels for Embryo Transfer. International Seminar on Camel Applied Research and Development. N.R.C.C. August 1998. Bikaner India. August 1998. J.C.P.R.

Tinson A. H., K. S. Kuhad, K. Singh, R. Sambayal, A. Mugheiry, A. Rahman and J. Al Masri, 2001. Twinning in Camels 4th Proc. Arid Zone Al-Ain Nov 2001 (Abstract).

Welshons W. J. L. B. and Russell, 1959. The $\mathrm{Y}$ chromosome as the bearer of male determining factors in the mouse. Proc Natl. Acad. Sci. USA 45: 560-566 